\title{
Mobility of Polyethylene Glycol-Modified Urethane Acrylate (PMUA) Nanoparticles in Soils
}

\author{
Warapong Tungittiplakorn ${ }^{1 *}$, Viranart Kongbua $^{1}$, Anyamanee Tulaphan ${ }^{1}$, and Kannika Kaewtawee ${ }^{1}$ \\ ${ }^{1}$ King Mongkut's University of Technology North Bangkok, Thailand
}

\begin{abstract}
Engineered nanoparticles (ENPs) have been reported for their potential to enhance in situ soil remediation due to their size and stability in water. These properties allow them to pass through soils with minimal loss in soil flushing or pump-and-treat process. The success of nanoparticle-facilitated soil flushing depends on the mobility of nanoparticles in the soil matrix. However, organic carbon content and soil texture can affect the mobility of nanoparticles in soils. This study compared the mobility of polyethylene glycolmodified urethane acrylate (PMUA) nanoparticles in three types of soils with varying organic contents. The results of two consecutive injection experiments showed that the recovery of injected nanoparticles through a soil column were 91 and $97 \%$ for sandy soil with carbon content of $0.01 \%, 81$ and $85 \%$ for clay loam soil with organic carbon content of $1.20 \%$ and 67 and $73 \%$ for clay soil with organic carbon content of $3.25 \%$. Furthermore, the batch experiments showed that the distribution coefficient $(\mathrm{Kd})$ of PMUA nanoparticles between water and sandy soil, clay loam soil, and clay soil were $1.86,2.34$ and $3.01 \mathrm{~mL} / \mathrm{g}$, respectively. This conforms to the column experiment results and confirms that the increase in organic carbon content in soils increases the adsorption of PMUA nanoparticles, and therefore decreases the mobility of the nanoparticles through soils. Moreover, the distribution coefficient from batch experiments could be used to predict the mobility of PMUA nanoparticles in soils, and the viability of in situ PMUA-facilitated soil flushing method for specific contaminated soils.
\end{abstract}

\section{Introduction}

Contamination of hydrophobic organic pollutants, such as polycyclic aromatic hydrocarbons (PAHs) and polychlorinated biphenyls (PCBs) have been reported worldwide. Because of their hydrophobic properties, PAHs and PCBs can sorb strongly to soils and persist in the environment for a long time. They can also enter the food chains and cause health issues in human including cancer [1]. One of the widely used remediation methods for these types of soil contaminants is in situ soil flushing or pump-and-treat method [2]. However, these contaminants are difficult to be flushed out from soils because of strong sorption. To increase the efficacy of soil flushing, surfactants have been applied to increase the apparent solubility of organic contaminants by micellar solubilzation [3]. The size of surfactant micelles are in nano-scale so they are able to mobilize the contaminants to the extraction well where contaminated groundwater is pumped up to be treated [4]. The limitation of this method is that for the micellar solubilization to occur, the concentration of the surfactant must exceed its critical micelle concentration (CMC), otherwise surfactant monomers would not be effective for solubilizing the contaminants. Moreover, surfactant micelles can easily break and sorb on the soils, thus limiting their use for mobilizing the contaminants [5].

In order to solve these problems, engineered nanoparticles (ENPs) have increasingly been studied [6].
Polyethylene glycol-modified urethane acrylate (PMUA) nanoparticle is a type of ENPs that was designed to have hydrophobic and hydrophilic moiety similar to that of non-ionic surfactants. The hydrophobic interior of the nanoparticles has high affinity for hydrophobic organic pollutants while the hydrophilic surface prevents aggregation and promotes particle mobility in soils. However, unlike surfactant micelle, the core of PMUA nanoparticles is chemically cross-linked so that they remain intact regardless of the concentration. Moreover their nanosize and steric stability (provided by PEG pendant chain) allows PMUA nanoparticles to travel through soil pores without much loss regardless of the ionic strength of the water. This resulted in excellent mobility of PMUA nanoparticles in soil as reported by Kim et. al. [7] and Tungittiplakorn et. al. [8]. Model simulations also showed the use of PMUA could still be effective in soils with higher organic carbon content [9].

However, all experiments in the aforementioned studies were performed on sandy soils with low organic carbon content $(0.049 \%$ [7] and $0.57 \%$ [8]) whereas natural soils may contain higher amount of silt and clay, with higher organic carbon content. The average organic carbon content of surface soils can be $6 \%$ [10]. As a result, natural soils can sorb organic chemicals strongly [11]. This may interfere with the mobility of PMUA in natural soil and affect the efficacy of PMUA nanoparticlefacilitated in situ soil flushing [9]. In this study we tried to prove whether PMUA nanoparticles are effective for

"Corresponding author: warapong.t@sci.kmutnb.ac.th 
enhancing in situ soil flushing by comparing the affinity and the mobility of PMUA nanoparticles in three types of soils with varying organic contents.

\section{Experimental methods}

\subsection{Materials}

Three soil samples were obtained from Bangkok, Uthai Thani, and Cha Choeng Sao provinces. The soil organic carbon contents (OC) were determined with the WalklyBlack method. The soil particle size distributions were determined by sieve analysis method and the soil textures were classified using the US Department of Agriculture method [12]. The properties of soil samples are presented in Table 1.

Table 1. Properties of soil samples.

\begin{tabular}{cccccc}
\hline $\begin{array}{c}\text { Soil } \\
\text { Sample }\end{array}$ & $\begin{array}{c}\text { OC } \\
(\%)\end{array}$ & $\begin{array}{c}\text { Sand } \\
(\%)\end{array}$ & $\begin{array}{c}\text { Silt } \\
(\%)\end{array}$ & $\begin{array}{c}\text { Clay } \\
(\%)\end{array}$ & $\begin{array}{c}\text { Soil } \\
\text { Texture }\end{array}$ \\
\hline 1 & 0.01 & 98 & 1 & 1 & $\begin{array}{c}\text { Sand } \\
\text { Clay }\end{array}$ \\
2 & 1.20 & 39 & 30 & 31 & $\begin{array}{c}\text { Loam } \\
\text { Clay }\end{array}$ \\
3 & 3.25 & 26 & 26 & 48 & \\
\hline
\end{tabular}

The chemicals used in the synthesis of PMUA nanoparticles were poly(tetramethylene glycol) (PTMG, MW = 1000), 2,4-toluenediisocyanate (TDI), 2-hydroxyethyl methacrylate (HEMA), and poly(ethylene glycol) (PEG, $\mathrm{MW}=2000$ ). N,N-dimethylacetamide (DMAc) was used as a solvent in the synthesis. Potassium persulfate (KPS) was used as an initiator for the crosslinking process. All chemicals were used as received from the provider (S.N.P General Trading Co., Ltd.).

\section{2 Synthesis of polyethylene glycol-modified urethane acrylate (PMUA) nanoparticles}

PMUA nanoparticles were synthesized using the method described by Tungittiplakorn et al. [8]. The molar ratio of PTMG, TDI, HEMA, and PEG was 1:2:1:1. The average size of the nanoparticles was measure by a dynamic light scattering system and found to be approximately $80 \mathrm{~nm}$.

\subsection{Batch Experiments}

The affinity of the nanoparticles and soils were measured by determining the distribution coefficients in batch sorption experiments. The distribution coefficients of nanoparticles between DDI water and soils $\left(\mathrm{K}_{\mathrm{d}}\right)$ were determined by mixing $1 \mathrm{~g}$ of soil sample with $10 \mathrm{~mL}$ of nanoparticle at different concentrations in $15 \mathrm{~mL}$ vials. The vials were shaken at $100 \mathrm{rev} / \mathrm{min}$ at room temperature (approximately $28^{\circ} \mathrm{C}$ ) for $24 \mathrm{~h}$. The supernatant was filtered with No.42 filter paper to separate the soil and sorbed nanoparticles from the liquid suspension. Three milliliter samples from the filtered supernatant were dried at $105^{\circ} \mathrm{C}$ for $3 \mathrm{~h}$ and desiccated for $1 \mathrm{~h}$. The dry weight was used to calculate the concentration of nanoparticles in the supernatant. All experiments were performed in triplicates.

\subsection{Column experiments}

Column experiments were performed to determine the mobility of PMUA nanoparticles in different types of soils. Soil samples were packed into a stainless steel column (2.54 cm in diameter and $10 \mathrm{~cm}$ in length). Nitrate was used as a nonreactive tracer to determine the pore water velocity. A UV-visible spectrophotometer (Thermo Fisher Scientific) was used to measure the nitrate concentration. The porosity of the column was determined from the weight of the column before and after saturating the pore space with water.

One pore volume of DDI water was injected into the column followed by 1 pore volume of nanoparticle suspension $\left(3 \mathrm{~g} / \mathrm{L}\right.$ ) with $0.02 \% \mathrm{NaN}_{3}$ (to prohibit microbial growth) and followed by 1 pore volume of DDI water. The flow rate was controlled by a peristaltic pump (Shenchen YZ1515x) at $20 \mathrm{~mL} / \mathrm{h}$. The experiment was performed twice for each soil sample. The effluent from the column was continuously collected in vials and measured for the nanoparticle concentration with the same method as described in the sorption experiment section.

\section{Results and discussion}

\subsection{Batch experiments}

The results of batch sorption experiments of PMUA nanoparticles on soils were presented in Fig. $1-3$. The isotherms showed that the sorption of nanoparticles on all soil samples followed linear or C-type isotherm [10]. This could be because the concentration of PMUA nanoparticles used in this study was quite low (less than $1.5 \mathrm{~g} / \mathrm{L}$ ) so that the sorption sites on the soil surface were not filled up by the nanoparticles.

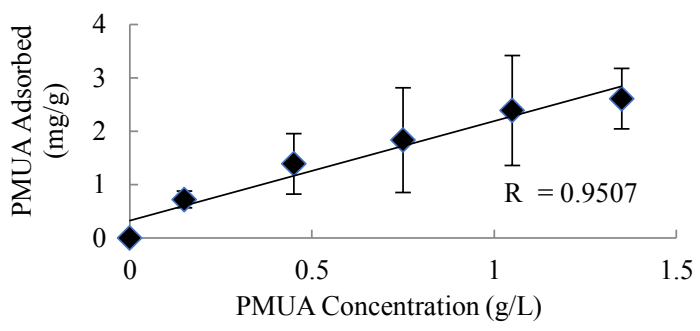

Fig. 1.Sorption of PMUA nanoparticle on sandy soil.

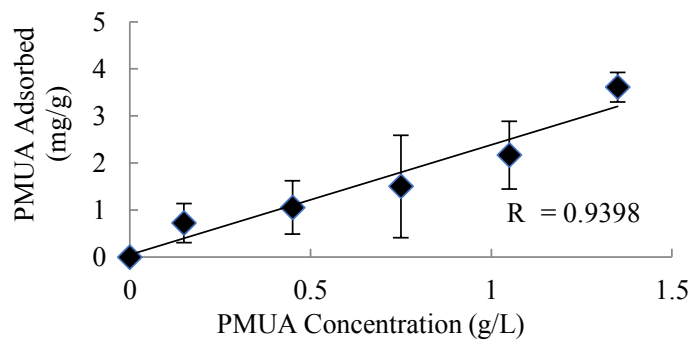

Fig. 2. Sorption of PMUA nanoparticle on clay loam soil. 


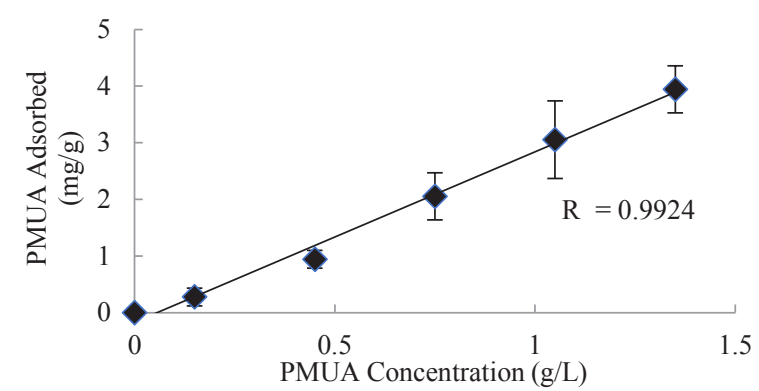

Fig. 3. Sorption of PMUA nanoparticle on clay soil.

The distribution coefficients $\left(\mathrm{K}_{\mathrm{d}}\right)$ of the nanoparticles between soil and DDI water with standard errors were calculated from the slope of the corresponding isotherm and summarized in Table 2. The distribution coefficient of PMUA nanoparticles between sandy soil and water was the lowest while the distribution coefficient of PMUA nanoparticles between clay soil and water is the highest. This shows that sorption of the nanoparticles on soils increase with the amount of organic carbon content in the soils.

Table 2. The distribution coefficients of nanoparticles between soils and DDI water and the organic carbon contents of soils.

\begin{tabular}{|c|c|c|}
\hline Soil Sample & $\mathbf{K}_{\mathbf{d}}(\mathbf{m L} / \mathbf{g})$ & OC $\mathbf{( \% )}$ \\
\hline Sand & $1.86 \pm 0.21$ & 0.01 \\
\hline Clay Loam & $2.34 \pm 0.30$ & 1.20 \\
\hline Clay & $3.01 \pm 0.13$ & 3.25 \\
\hline
\end{tabular}

When the distribution coefficients were plotted against the organic carbon contents as shown in Fig. 4, the relationship appeared to be linear with the correlation coefficient of 0.9984 . This is consistent with the linear partition theory which states that the distribution coefficient of organic substance is linearly correlated with the organic carbon content of the soil [13]. This shows that pendent water-soluble chains on the particle surface could not prevent PMUA nanoparticles from sorbing on soil surface. As a result, there might be more particle loss than previously expected when the nanoparticles are applied to natural soil with high fraction of silt and clay or organic carbon content. The results of column experiments presented in the next section would confirm the results of batch experiments.

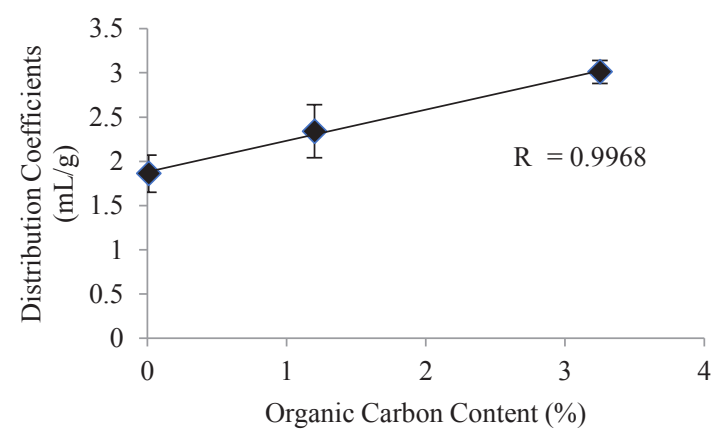

Fig. 4. The relationship between the distribution coefficients of PMUA nanoparticles between soils and DDI water and the organic carbon contents of the soils

\subsection{Column Experiments}

The breakthrough curves of PMUA nanoparticles for each soil sample are presented in Fig. $5-7 . \mathrm{C} / \mathrm{C}_{\mathrm{o}}$ represents the ratio of the concentration of the effluent to the influent. The number of pore volume represents the ratio of the volume of injected water to the absolute pore volume of the soil. The porosity of the soil columns was determined to be $0.40,0.48$, and 0.63 for the sandy soil, clay loam soil, and clay soil column, respectively. It is apparent from the breakthrough curves that PMUA nanoparticles travel at almost the same velocity as nitrate tracer which means that PMUA nanoparticles are extremely mobile in all types of soils used in this study.

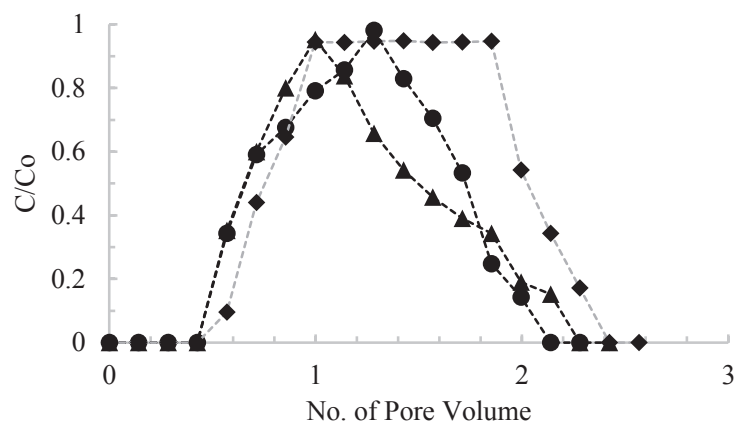

Fig. 5. Breakthrough curves of tracer $)\left(, 1^{\text {st }}\right.$ injection ) $\boldsymbol{\Delta}\left(\right.$, and $2^{\text {nd }}$ injection $) \bullet($ of PMUA nanoparticle in sandy soil column

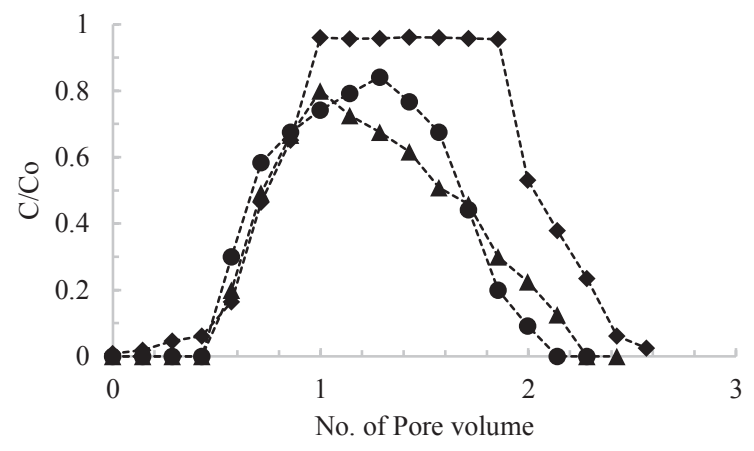

Fig. 6. Breakthrough curves of tracer $(\bullet), 1^{\text {st }}$ injection $(\Delta)$, and $2^{\text {nd }}$ injection $(\bullet)$ of PMUA nanoparticle in clay loam soil column.

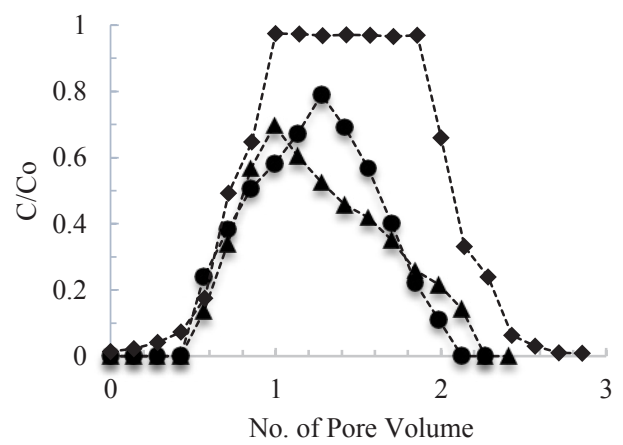

Fig. 7. Breakthrough curves of tracer $) \diamond\left(, 1^{\text {st }}\right.$ injection ) $\mathbf{\Delta}$ (, and $2^{\text {nd }}$ injection $) \bullet($ of PMUA nanoparticle in clay soil column. 
The mobility of PMUA nanoparticles in each type of soil was determined by calculating the recovery rate of nanoparticles that passed through the soil column. The recovery rates of PMUA and nitrate tracer are shown in Table 3. From the results in Table 3, the recovery of the first and second pulse of injected nanoparticles $(3 \mathrm{~g} / \mathrm{L})$ through soil column were 91 and $97 \%$ for sandy soil, 81 and $85 \%$ for clay loam soil, and 67 and $73 \%$ for clay soil. The second injection of nanoparticles resulted in higher recovery rate for all types of soil. This was because the soil surface sorbed some amount of nanoparticles in the first injection; therefore less sorption sites were available for the second injection, allowing more particles to pass through.

Table 3. Recovery rates of PMUA nanoparticles through soil columns.

\begin{tabular}{|c|c|c|c|}
\hline \multirow[b]{2}{*}{$\begin{array}{c}\text { Type of } \\
\text { Soil }\end{array}$} & \multicolumn{3}{|c|}{ Recovery Rate (\%) } \\
\hline & $\begin{array}{c}\text { PMUA } \\
\text { Nanoparticles } \\
*\end{array}$ & $\begin{array}{c}\text { PMUA } \\
\text { Nanoparticles } \\
* * *\end{array}$ & Tracer \\
\hline Sand & 91 & 97 & 100 \\
\hline $\begin{array}{l}\text { Clay } \\
\text { Loam }\end{array}$ & 81 & 85 & 100 \\
\hline Clay & 67 & 73 & 100 \\
\hline
\end{tabular}

$* 1^{\text {st }}$ Injection $* * 2^{\text {nd }}$ Injection

These results are consistent with the results reported by Tungittiplakorn et al. [8]. However, the concentration of PMUA nanoparticles used in their experiments was 5 times higher $(15 \mathrm{~g} / \mathrm{L})$ resulting in much less particle recovery for the first injection $(38 \%)$ and complete recovery $(100 \%)$ for the second injection because all sorption sites were filled up by the first injection. The experimental results of this study clearly indicated that the mobility of PMUA nanoparticles in soil columns decreased with the increase in soil organic carbon content.

Transport of particles through porous media depends on many factors such as hydraulic conductivity and interactions of the particles with the media. Clay is known to have very high surface area and numerous micropores. High surface area causes clay particles to sorb large amount of solutes in the water including organic matter. Soil organic carbon (SOC) is highly correlated to the extent of compound sorption [10]. This caused clay loam and clay soil which contained higher organic carbon to sorb PMUA nanoparticles more than sandy soils. Sorption and accumulation of nanoparticles on the soil surface could cause clogging in the soil pores and change the permeability and hydraulic conductivity of the soil [14]. Micropores in clay particles could have trapped nanoparticles and lead to irreversible loss of nanoparticles. Aggregation could also affect particle transport through porous media $[15,16]$ but previous study showed that PMUA nanoparticles did not coagulate or change in particle size regardless of the ionic strength or the presence of cations [8], therefore this should not have been the cause of particle loss in this study. Flow rate is another factor that influences particle mobility in porous media. The increase in flow rate improves the elution and recovery of the particles [17]. However, the flow rate used in this study is the same or similar to other studies (approximately $20 \mathrm{~mL} / \mathrm{h}$ ) in order to simulate real groundwater flow.

Despite the fact that PMUA nanoparticles used in this study sorbed on soil with high organic carbon content, the chemical structure of the nanoparticles could be improved by varying the molar ratio or the size of PEG used in the polymer synthesis to improve its stability and the ability to prevent sorption on soil surface.

\section{Conclusion}

The experimental results showed that even though PMUA nanoparticles were designed to have PEG pendent chain that inhibits the aggregation and the sorption of nanoparticles on soil surface and the chemically crosslinked microstructure of nanoparticles that prevent them from breaking up and being sorbed on soil surface [7, 8], their mobility in natural soil with high organic content may still be reduced significantly. The viability of nanoparticle-facilitated in situ soil flushing depends on the ability of nanoparticles to compete with soil in sorbing organic pollutants and the mobility of nanoparticles to travel through soil without being loss in the soil matrix. When considering the use of PMUA nanoparticles for soil remediation in a particular soil, the distribution coefficient from batch experiments could be used to predict the mobility of nanoparticles in the soil as they correlate well with the recovery of the particles.

PMUA nanoparticles have been shown to have superior ability in absorbing organic pollutants, but the fact that they can significantly sorb on soil with high organic carbon content may limit their use as an alternative to surfactants. Losing nanoparticles in the soil means that the cost of remediation will be higher as the nanoparticles cannot be recovered and reused. Moreover, there has been an emerging concern about microplastic and nanoparticles persisting in the environment. These particles may be toxic to the ecosystem and human health when they enter food chains [18, 19]. If PMUA nanoparticles are trapped in the aquifer, they may become another source of new pollutants. At present, ecotoxicity of PMUA nanoparticles is unknown; therefore the use of PMUA nanoparticles for soil remediation must be studied further and applied with careful consideration.

This research was funded by King Mongkut's University of Technology North Bangkok. Contract no. KMUTNB-NEW-57-04.

\section{References}

1. N. Rodriguez-Eugenio, M. McLaughlin, D. Pennock, Soil Pollution: a Hidden Reality (2018)

2. D.A. Edwards, R.G. Luthy, Z. Liu, Environ Sci Technol. 25 (1991)

3. D.A. Sabatini, R.C. Knox, J.H. Harwell, J Am Chem Soc. 594, (1995)

4. A. Karthick, B. Roy, P. Chattopadhyay, J Environ Manage. 243 (2019)

5. J.-Y. Kim, C. Cohen, M.L. Shuler, L.W. Lion, Environ Sci Technol. 34 (2000) 
6. Q. Li, X. Chen, J. Zhuang, X. Chen, Environ Sci Pollut Res. 23, 12 (2016)

7. J.-Y. Kim, S.-B. Shim, J.-K. Shim, J Hazard Mater. $98(2003)$

8. W. Tungittiplakorn, L.W. Lion, C. Cohen, J.-Y. Kim, Environ Sci Technol. 38 (2004)

9. W.L.W. Johari, P.J. Diamessis, L.W. Lion, Water Research. 44, 4 (2010)

10. M.E. Essington, Soil and water chemistry : an integrative approach (2004)

11. W. Lertpaitoonpan, S.K. Ong, T.B. Moorman, Chemosphere 76, 4 (2009)

12. M. van der Perk, Soil and Water Contamination, 2nd Edition (2013)
13. D.L. Sparks, Environmental Soil Chemistry (Second Edition) 133-186 (2003)

14. M.A. Endo Kokubun, A. Muntean, F.A. Radu, K. Kumar, I.S. Pop, E. Keilegavlen, K. Spildo, Chem Eng Sci. 207, (2019)

15. A.R. Esfahani, A.F. Firouzi, G. Sayyad, A.R. Kiasat, J Ind Eng Chem. 20, 5 (2014)

16. A. Taghavy, K.D. Pennell, L.M. Abriola, J Contam Hydrol. 172 (2015)

17. I. Chowdhury, Y. Hong, R.J. Honda, S.L. Walker, J. Colloid Interface Sci. 360, 2 (2011)

18. N.B. Turan, H.S. Erkan, G.O. Engin, M.S. Bilgili, Process Saf Environ. 130 (2019)

19. X. Huangfu, Y. Xu, C. Liu, Q. He, J. Ma, C. Ma, R. Huang, Chemosphere 219 (2019) 